\title{
Al in endoscopy: is the computer innocent in case of missed cancer?
}

\section{두)(요우}

\author{
Authors \\ Cesare Hassan ${ }^{1}$, Giulio Antonelli ${ }^{1}$, Mario Dinis-Ribeiro ${ }^{2}$ \\ Institutions \\ 1 Endoscopy Unit, Nuovo Regina Margherita Hospital, \\ Rome, Italy \\ 2 CIDES/CINTESIS, Faculty of Medicine, University of \\ Porto, Porto, Portugal
}

Bibliography

Endoscopy International Open 2020; 08: E1387-E1388

DOI 10.1055/a-1214-5937

ISSN 2364-3722

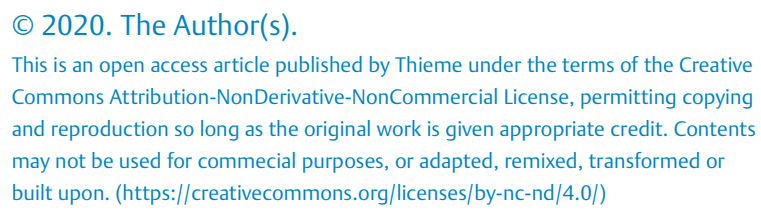

Corresponding author

Cesare Hassan, ONRM Hospital - Gastro, Via Morosini 30

Rome 00153, Italy

Fax: +390658446533

cesareh@hotmail.com
While doing a screening colonoscopy, the possibility of missing a lesion crosses our minds from time to time, especially after a negative procedure in which we failed to detect even a sub-centimeter adenoma. We then disregard this strange idea as a bad nightmare, while continuing our screening procedures. Don't we deceive ourselves pretending that interval colorectal cancer (CRC) is not an issue, or if it is an issue, consider that the responsibility for it falls to other endoscopists who are much less skillful than us?

Irrespective of an endoscopist's competence and dedication, interval CRC inevitably occurs. Updated evidence suggests that $1 \%$ of our patients will suffer from an interval CRC every 10 years [1,2]. If I do 1000 colonoscopy per year, 10 of those patients will come back to me within that time frame with a CRC that I was expected to prevent.

It is hard to argue against the crude belief of an unlucky patient that we are guilty rather than innocent in not diagnosing interval CRC. It is the epidemiological evidence that condemns us by consistently showing that nearly all interval CRC comes from missed lesions [3]. This is worsened by the parallel evidence that a $25 \%$ to $30 \%$ miss rate for colorectal neoplasia at screening colonoscopy has been widely reported, unexpectedly even in expert centers. If we miss polyps, we cause the cancer, so what's wrong with the straightforward conclusion of our patients?

Despite the logic in our patients' assumption, it appears a bit unfair to us as endoscopists. We spend several hours every day in an extremely boring screening activity by filtering through our mind over 50,000 photographs per colonoscopy, totaling a half million frames per 10 colonoscopies [4]. Most polyps, even those advanced, are present on only a few frames, sometimes in a very peripheral part of the screen. To demand infallibility is out of this world. It is undeniable that we are excessively penalized by the burden of responsibility for any unprevented CRC, especially when considering that it represents disproportionally little harm when compared with the substantial benefit that we provide.

After several years of resignation about this unsolvable dilemma, artificial Intelligence (AI) came to save us! Isn't it true that any single frame is now screened by a super-accurate computer? Isn't it true that for any overlooked lesion, there will be a visual and acoustic alarm to wake us from any distraction, tiredness, or mere incompetence? What was a sub-centimeter flat lesion with subtle contrast from the surrounding mucosa in the remarkable width of an over-insufflated proximal colon is now suddenly replaced by a big, well-delineated, full-color Al box accompanied by a pleasurable sound, reminding us of our unceasing human duty and responsibility! No doubt, it is much easier to detect a box than a lesion! This is fully confirmed in this issue of Endoscopy International Open by the enthusiasm shown by US endoscopists over the potential efficacy of Al in improving the detection rate. The risk for being blamed for an interval cancer after a negative colonoscopy is over, it will be remembered as prehistory of screening colonoscopy!

Unfortunately, there are two postulates in Al that undermine such overzealous optimism. First, what engineers name as "deep learning" is a series of algorithms automatically derived by the computer after supervised training with annotated lesions. Nobody knows how these algorithms work, incorporating unpredictability in what was a fully predictable screening procedure. For instance, despite its accuracy in detecting subcentimeter lesions, Al may miss a 3-cm deeply invasive cancer 
for the simple reason that no training dataset has enough cases of relatively rare lesions to appropriately train the system [5]. What is true for a large cancer may also apply to more subtle lesions, such as non-granular lateral spreading tumors (LSTs), sessile serrated lesions with dysplasia, or plaque-like early CRC. Second, the human endoscopist still must discriminate between a true- and a false-positive Al finding. Thus, Al may correctly identify a subtle lesion, but the poor endoscopist may discard it as false-positive and feel twice as guilty about having missed the lesion in the first place and then discarding it when appropriately presented by the Al system.

If we cannot fully predict Al incorporation in clinical practice, it is much easier to predict human nature. Any time a patient sues the endoscopist for a missed cancer, the endoscopist is likely to sue the developers of the Al software for not having alerted to the overlooked lesion. Who is to be blamed-the endoscopist, the software house, the health system employing either or both of them, or all of them together?

Legally speaking, each party must show that they have adhered to a reasonable professional standard based on the assumption that in the same or similar circumstances, any professional would have make the same decisions, based on competence and medical or technical knowledge. For instance, any health system may be found reasonably guilty if no measure to monitor key quality indicators, such as adenoma detection rate or adequate documentation of the procedure, was in place. Similarly, an endoscopist is expected to prove his or her competence, irrespective of $\mathrm{Al}$, as $\mathrm{Al}$ only assists in diagnosis, as exemplified in the acronym CAD (computer-aided diagnosis) [6]. In other words, if I miss a non-granular LST with already invasive cancer that any expert endoscopist should detect, I remain responsible, irrespective of whether the lesion was detected by $\mathrm{Al}$.

What about the computer? Is it always innocent only because we as clinicians are primarily responsible for the clinical outcome of a diagnostic procedure? Imagine a similar humanmachine interaction with a different scenario. A pilot is flying in a storm. He intends to accelerate, but the computer mistakenly advises him to decelerate. He trusts the computer more than his own judgment, and the airplane crashes. Later, the software in the onboard computer is found to have contained severe mistakes, which prevented the pilot from making the correct choice. The fact that the endoscopist is ultimately responsible for patient outcome does not mean that the interaction between the computer and the human mind is irrelevant. It is a given that the endoscopist will, in one way or another rely on the computer input based on the assumption that the computer is doing its job well. On the other hand, if the software is corrupted or provides incomplete information, that human trust has been somewhat betrayed, and the engineer is likely to be just as or more liable than the endoscopist.

The fact that the computer can be the guilty actor in litigation about an interval CRC should not be misjudged as good news for the endoscopy community! On the other hand, it poses additional responsibility over us as endoscopists. Introducing Al into our practice does not simply mean buying an Al system and switching it on. We have a moral responsibility to become fully informed about how the system was trained (i.e, how many lesions and of what size, morphology, location, and histology), how robust the data are on the Al system's standalone performance, what the likelihood is of each output being a true- or false-positive finding, and its outcome in a clinical setting. In addition, as already mentioned, we must be fully competent in not rejecting a true positive because of incompetence in recognizing an Al-detected subtle neoplasia. This is well defined in the recent guideline from the European Society of Gastrointestinal Endoscopy (ESGE), which states that only Al systems with robust clinical data should be used in endoscopy practice [6].

\section{Conclusion}

In conclusion, Al may be a terrific support for endoscopists in preventing catastrophic misses of colorectal or other neoplasia at screening endoscopy. However, it should not be considered as a mere simplification of our standard, but rather, as an ineludible increase in complexity that require extensive knowledge of Al technology and adequate training in lesion recognition.

\section{Competing interests}

The authors declare that they have no conflict of interest.

References

[1] Corley DA, Jensen CD, Marks AR et al. Adenoma detection rate and risk of colorectal cancer and death. N Engl J Med 2014; 370: 12981306

[2] Rutter MD, Beintaris I, Valori R et al. World Endoscopy Organization Consensus Statements on Post-Colonoscopy and Post-Imaging Colorectal Cancer. Gastroenterology 2018; 155: 909-925.e3

[3] Zhao S, Wang S, Pan P et al. Magnitude, risk factors, and factors associated with adenoma miss rate of tandem colonoscopy: a systematic review and meta-analysis. Gastroenterology 2019; 156: 1661-1674.e11

[4] Repici A, Badalamenti M, Maselli R et al. Efficacy of real-time computer-aided detection of colorectal neoplasia in a randomized trial. Gastroenterology 2020: doi:10.1053/j.gastro.2020.04.062

[5] Hassan C, Wallace MB, Sharma P et al. New artificial intelligence system: first validation study versus experienced endoscopists for colorectal polyp detection. Gut 2020; 69: 799-800

[6] Bisschops R, East JE, Hassan C et al. Advanced imaging for detection and differentiation of colorectal neoplasia: European Society of Gastrointestinal Endoscopy (ESGE) Guideline - Update 2019. Endoscopy 2019; 51: 1155-1179 